\title{
Positron Production by X Rays Emitted by Betatron Motion in a Plasma Wiggler
}

\author{
D. K. Johnson, ${ }^{1}$ D. Auerbach, ${ }^{1}$ I. Blumenfeld, ${ }^{2}$ C. D. Barnes, ${ }^{2}$ C. E. Clayton, ${ }^{1}$ F. J. Decker, ${ }^{2}$ S. Deng, ${ }^{3}$ P. Emma, ${ }^{2}$ \\ M. J. Hogan, ${ }^{2}$ C. Huang, ${ }^{1}$ R. Ischebeck, ${ }^{2}$ R. Iverson, ${ }^{2}$ C. Joshi, ${ }^{1}$ T. C. Katsouleas, ${ }^{3}$ N. Kirby, ${ }^{2}$ P. Krejcik, ${ }^{2}$ W. Lu, ${ }^{1}$ \\ K. A. Marsh, ${ }^{1}$ W. B. Mori, ${ }^{1}$ P. Muggli, ${ }^{3}$ C. L. O'Connell, ${ }^{2}$ E. Oz, ${ }^{3}$ R. H. Siemann, ${ }^{2}$ D. Walz, ${ }^{2}$ and M. Zhou ${ }^{1}$ \\ ${ }^{1}$ University of California, Los Angeles, California 90095, USA \\ ${ }^{2}$ Stanford Linear Accelerator Center, Stanford, California 94309, USA \\ ${ }^{3}$ University of Southern California, Los Angeles, California 90089, USA
}

\begin{abstract}
Positrons in the energy range of $3-30 \mathrm{MeV}$, produced by $\mathrm{x}$ rays emitted by betatron motion in a plasma wiggler of $28.5 \mathrm{GeV}$ electrons from the SLAC accelerator, have been measured. The extremely highstrength plasma wiggler is an ion column induced by the electron beam as it propagates through and ionizes dense lithium vapor. $\mathrm{X}$ rays in the range of $1-50 \mathrm{MeV}$ in a forward cone angle of $0.1 \mathrm{mrad}$ collide with a $1.7 \mathrm{~mm}$ thick tungsten target to produce electron-positron pairs. The positron spectra are found to be strongly influenced by the plasma density and length as well as the electron bunch length. By characterizing the beam propagation in the ion column these influences are quantified and result in excellent agreement between the measured and calculated positron spectra.
\end{abstract}

High-energy physicists use electron-positron collisions to validate the predictions of various field theories. The positrons $\left(e^{+}\right)$needed for the collisions are currently produced by bombarding a high- $Z$, solid target that is several radiation lengths $\left(X_{0}\right)$ thick with a high-energy electron beam [1]. The resulting interaction creates bremsstrahlung $\mathrm{x}$-ray photons which can interact with the atomic nuclei of the target producing electron-positron pairs. These currently used "thick-target", bremsstrahlung $e^{+}$sources may well fail when scaled to meet the requirements of future linear colliders, due to thermal stress fatigue from large volumetric $e^{-}$beam energy deposition into the target from ionization and multiple scattering. One way to reduce this problem is to produce the $\mathrm{x}$ rays separately from a thin $\left(<X_{0}\right)$, high- $Z$ target where the $\mathrm{x}$-ray conversion to $e^{+}-e^{-}$ pairs takes place. Several such alternative designs for next generation $e^{+}$sources, including the use of a helical undulator or Compton backscattering off a circularly polarized laser beam, to produce polarized photons in the 5$10 \mathrm{MeV}$ range [2,3], are being studied. It is imperative that any proposed source have a high conversion efficiency of drive beam energy into $\mathrm{x}$-ray photons in the tens of $\mathrm{MeV}$ range for efficient $e^{+}$production [4]. In this Letter, we demonstrate the basic principles and scaling laws for generating positrons from a thin target via a novel scheme: using X-rays emitted by betatron motion of high-energy electrons in a high-strength plasma wiggler [5-7].

The plasma wiggler in this scheme is an ion column produced by an electron beam as it propagates through a column of lithium $(\mathrm{Li})$ vapor. If the beam spot size at the $\mathrm{Li}$ column entrance is small enough or the current density is high enough, the transverse electrostatic field of the beam completely field ionizes the $\mathrm{Li}$ vapor [8,9], creating a singly ionized plasma during the rise time of the electron beam. If the beam density $n_{b}$ is greater than the plasma density $n_{p}$, the space charge force of the beam rapidly expels the plasma electrons and creates a pure ion column $\left(n_{p}=n_{i}\right)$. The focusing force $-e E_{r}$ of this ion column causes the beam electrons to oscillate [10] and emit betatron $\mathrm{x}$ rays. This ion column also exerts a restoring force on the expelled plasma electrons which rush back, overshoot the ion column axis, and set up a wake oscillation and thus a longitudinal wakefield. In addition to radiating some of their energy due to betatron oscillations, the electrons can lose energy because of the retarding effect of this wakefield [11].

In previous work [6] the use of such a plasma wiggler was demonstrated for the generation of an intense collimated $x$-ray beam in the $1-10 \mathrm{keV}$ range. In the present work, the same basic concept has been extended for generating photons in the $1-50 \mathrm{MeV}$ range using a much higher density plasma and much shorter electron pulses. Whereas in [6] the energy loss by the electrons to both the wake and betatron $\mathrm{x}$-ray emission was a small perturbation, in the present work it is a significant fraction of its initial energy. This energy loss, beam-plasma ionization, and focusing effects must be properly accounted for in this work to get a quantitative agreement between the measured and calculated $e^{+}$spectra.

The radial electrostatic field of the ion column-the plasma wiggler-is given by $E_{r}(r)=\left(n_{p} e / 2 \epsilon_{o}\right) r$ (SI units) $[7,10]$. The fundamental parameter describing the plasma wiggler is the wiggler strength defined as $K=$ $\gamma_{b} k_{\beta} r_{o}$, where $k_{\beta}=\omega_{p} / c \sqrt{2 \gamma_{b}}$ is the betatron wave number, $\omega_{p}$ is the plasma frequency, $\gamma_{b}$ is the electron Lorentz factor, and $r_{o}$ is the maximum radius of the oscillating electron [7]. Note that the electrons in a beam will have a distribution of $r_{o}$. The spectrum of the betatron radiation has frequencies $\omega_{n}=\frac{n 2 \omega_{\beta} \gamma^{2}}{1+K^{2} / 2+\left(\gamma_{b} \theta\right)^{2}}$, where $\omega_{\beta}=k_{\beta} c$ is 
the betatron frequency, $\theta$ is the angle between the axial motion of the beam electron and the observation point, and $n$ is the harmonic number [6,7]. When $K \gg 1$, high harmonic radiation dominates the spectrum, and since $K$ is linearly proportional to $r_{o}$, individual electrons at different radii have different radiated frequencies resulting in a broadband spectrum. This spectrum is characterized by the critical frequency $\omega_{c}=3 K \gamma^{2} \omega_{\beta} / 2$ (for on-axis radiation, $\theta=0$ ) and a critical harmonic number $n_{c}=3 K^{3} / 8$. $[6,7]$.

The electron energy loss to radiation is given by the relativistic Larmor formula. Using the betatron orbits due to $E_{r}$, the energy loss per unit length is

$$
\frac{d W_{\mathrm{loss}}}{d z}=\frac{1}{3} r_{e} m_{e} \gamma_{b}^{2} \omega_{\beta}^{2} K^{2} \propto\left(\gamma_{b}^{2}, n_{p}^{2}, r_{o}^{2}\right),
$$

where $m_{e}$ is the electron mass and $r_{e}$ is the classical electron radius $[6,7]$. As an example, in an ion column of density $n_{i}=3 \times 10^{17} \mathrm{~cm}^{-3}$, an electron with an energy of $28.5 \mathrm{GeV}$ and an $r_{o}$ of $10 \mu \mathrm{m}$ experiences a wiggler strength $K$ of 173 with an on-axis critical photon energy of roughly $50 \mathrm{MeV}$, ideal for $e^{+}$production. Equation (1) shows that this electron radiates $\sim 4.3 \mathrm{GeV} / \mathrm{m}$. Thus, such a high- $K$, meter-scale plasma wiggler is well suited for converting the electron's energy into a large number of multi-MeV photons, a necessary condition for a practical $e^{+}$source. We note, however, that for a group of electrons with a bunch length $\sim$ half a plasma wavelength, the electron energy loss to the wakefield can be far greater than that to radiation. Consequently, $\gamma_{b}$ changes continuously along its path through the plasma. If the plasma is long enough, the drop in $\gamma_{b}$ can be so severe that $\hbar \omega_{c}$ drops below $1 \mathrm{MeV}$ - the pair-production threshold.

The experiment [12] was carried out at the Final Focus Test Beam Facility (FFTB) at the Stanford Linear Accelerator Center (SLAC). A schematic of the experimental setup is shown in Fig. 1. A $28.5 \mathrm{GeV}$ beam containing approximately $N_{\text {bo }}=1.7 \times 10^{10}$ electrons was focused into a $\mathrm{Li}$ vapor having a variable length of $10-$ $30 \mathrm{~cm}$ and a variable neutral density of $n_{o}=1-30 \times$ $10^{16} \mathrm{~cm}^{-3}$. The focused beam has emittances $\epsilon_{x} \approx 5 \epsilon_{y} \approx$ $2.0 \times 10^{-9} \mathrm{~m}$-rad and a spot size of $\sigma_{x} \approx \sigma_{y} \approx 10 \mu \mathrm{m}$. Coherent transition radiation (CTR) in the $\mathrm{THz}$ range was detected after the bunch propagated through a $1 \mu \mathrm{m}$ thick Ti foil upstream of the plasma (not shown). The CTR energy $U_{\mathrm{CTR}}$ was measured using a pyroelectric detector. For a fixed $N_{\text {bo }}, U_{\mathrm{CTR}}$ is correlated to the inverse pulse length $\left(1 / \sigma_{z}\right)$ of the electron beam $[12,13]$, and ultimately to the wake amplitude. For $U_{\mathrm{CTR}}=400(\mathrm{AU}), \sigma_{z}$ is $\sim 20 \mu \mathrm{m}$ and, for the coarse of this work, $100 \leq U_{\mathrm{CTR}} \leq$ 500 .

The radiated betatron $\mathrm{x}$ rays propagate in vacuum $40 \mathrm{~m}$ downstream to the $e^{+}-e^{-}$pair convertor target. To minimize potential background signals on the $e^{+}$detectors, the maximum photon angular divergence of $\theta_{\max }=$
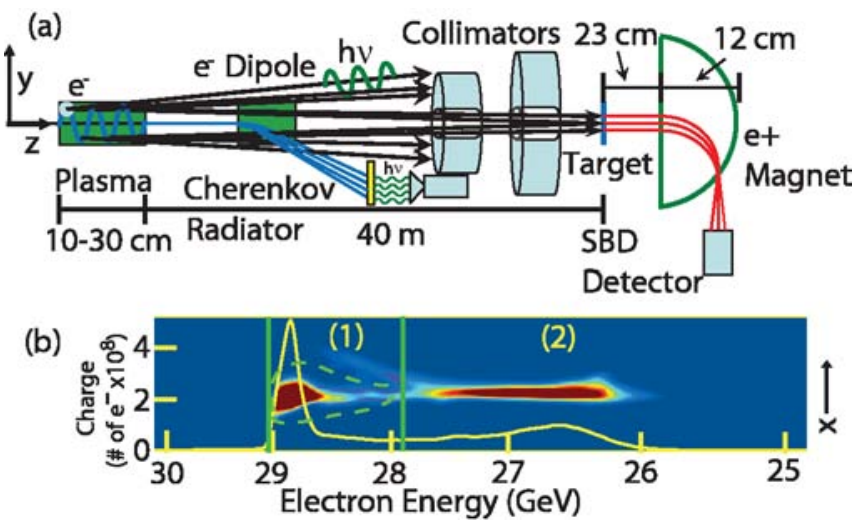

FIG. 1 (color). (a) An experimental schematic. (b) A typical electron energy spectrum image for $n_{p}=1 \times 10^{17} \mathrm{~cm}^{-3}$ and a plasma length of $11 \mathrm{~cm}$. A summed (over $x$ ) lineout of the spectrum is also shown (yellow solid line). In the absence of a plasma, the electron beam spectrum (dotted contour) is in the region of the spectrum labeled (1). Any beam electrons that lose energy will appear in region of the spectrum labeled (2).

$K_{\max } / \gamma_{b} \approx 9 \operatorname{mrad}$ was collimated down to $0.1 \mathrm{mrad}$ using two $10 \mathrm{~cm}$ long tungsten $(W)$ collimators with diameters of $6.4 \mathrm{~mm}$ and $12.7 \mathrm{~mm}$, located $25 \mathrm{~m}$ and $30 \mathrm{~m}$ from the plasma, respectively. These created an $8 \mathrm{~mm}$ collimated photon beam at the $1.7 \mathrm{~mm}\left(\approx 0.5 X_{0}\right)$ thick $W$ target producing $e^{+}-e^{-}$pairs. The resulting positrons (up to an energy of $30 \mathrm{MeV}$ ) were imaged by a magnetic spectrometer collecting a solid angle of $\approx 7 \times 10^{-3}$ sr and detected using $1 \mathrm{~mm}$ thick silicon surface barrier detectors (SBDs) with a surface area of $49 \mathrm{~mm}^{2}$. The electron beam exiting the plasma was deflected vertically by a dipole magnet to separate it from the $\mathrm{x}$ rays. It was subsequently imaged onto a Cherenkov radiator to record the spectrum of the beam electrons. An example of one such spectrum is shown in Fig. 1(b). Such images were used to estimate the number of electrons that interact with the ion column and their energies, as discussed later.

For each plasma density studied, the radiated x-ray energy, and therefore the $e^{+}$signal, was first optimized by adjusting the position of the beam waist with respect to the Li-column entrance. For instance, for $n_{p}=$ $1 \times 10^{17} \mathrm{~cm}^{-3}$, the optimum beam waist location was found to be at an axial position of $\sim-5 \mathrm{~cm}$ (at $\sim 10 \%$ of the peak Li density) as illustrated in Fig. 2(a). As the beam enters the neutral Li gas, whose measured profile is shown by the dotted green line in Fig. 2(a), the Li is ionized creating a low density plasma. This plasma acts as a thick lens that rapidly focuses the beam to a spot size smaller than its known vacuum value (dashed red line) before it begins betatron oscillations within the high density body of the plasma column (solid blue line) [14]. Once in this region, the beam envelope oscillates with a maximum $\sigma_{x, y} \simeq 4 \mu \mathrm{m}$.

Figure 2(b) shows the experimentally measured $e^{+}$ spectra for three different plasma densities. The spectrum 


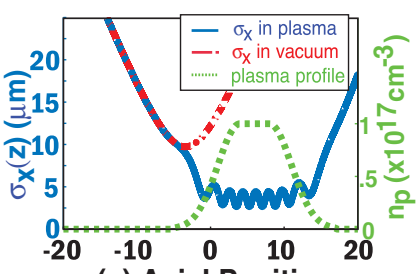

(a) Axial Position

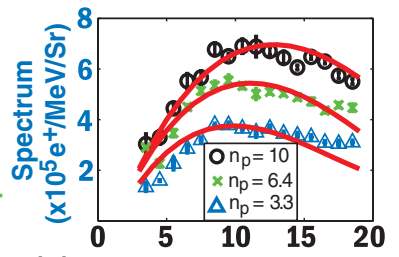

(b) Positron Energy (MeV)
FIG. 2 (color). (a) The calculated variation of $\sigma_{x}$ in vacuum (red dashed line) and inside the plasma (blue solid line) using a beam envelope model. The green dotted curve is the measured lithium vapor-density profile. (b) Measured and calculated $e^{+}$ spectra for three densities. The values of $n_{p}$ in the legend are in units of $10^{16} \mathrm{~cm}^{-3}$.

for $n_{p}=3.3 \times 10^{16} \mathrm{~cm}^{-3}$ peaks at $\sim 8 \mathrm{MeV}$ while that for $n_{p}=1.0 \times 10^{17} \mathrm{~cm}^{-3}$ peaks at a higher energy and has a higher yield as well. By changing the polarity of the magnet, the electron spectrum was also measured. The absolute yield and spectral shape were similar to that of the positrons, as expected from $e^{+}-e^{-}$pair production in the convertor target. Also plotted in Fig. 2(b) are the $e^{+}$ spectra (red curves) obtained from the calculated $\mathrm{x}$-ray spectra, as discussed in detail later. The measured $e^{+}$ spectra cover the energy range of interest for $e^{+}$sources for future linear colliders.

The radiation spectrum from the oscillating electrons in a plasma wiggler was computed using the formalism described in Ref. [15], which is well suited for a high- $K$ wiggler. When $K \gg 1$, only certain phases along the betatron trajectory contribute to the observed spectrum in the far field. These phases correspond to the maximum positive and negative displacement regions of the electron trajectory where the acceleration is the greatest and, locally, the momentum vector of the electron is pointing towards the converter target in the far field. By Taylor expanding the electron orbit around each of these contributing phases or "saddle points" [15] we calculate the total spectrum as the sum of the "synchrotronlike" bursts at each saddle point. The result gives the energy per unit frequency per unit solid angle $\left(\frac{d^{2} W}{d \omega d \Omega}\right)$. The $\mathrm{x}$-ray spectrum as a function of position is then input into the Monte-Carlo code Electron-Gamma Shower 4 (EGS4). Within EGS4, the photons collide with the $W$ target generating, among other things, the $e^{+}-e^{-}$ pairs. The positrons emanating from the rear of the target are propagated through the appropriate magnetic transport matrices to the locations of the SBDs.

By integrating the $e^{+}$spectra of Fig. 2(b) over energy, we can plot the $e^{+}$yield versus $n_{p}$ as shown in Fig. 3(a). According to Eq. (1), the total radiated photon energy should scale as $n_{p}^{2}$, as was observed in Ref. [6] where the maximum $n_{p}$ used was $\sim 600 \times$ lower than in this work. Since the critical energy $\hbar \omega_{c}$ for $3.3 \times 10^{16} \leq n_{p} \leq$ $1.0 \times 10^{17}$ - the $n_{p}$ range in Fig. $2(\mathrm{~b})$ - is $5.6 \mathrm{MeV} \leq$ $\hbar \omega_{c} \leq 17 \mathrm{MeV}$, the vast majority of the total radiated

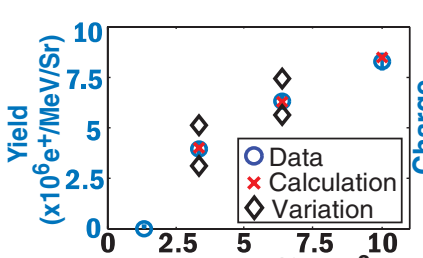

(a) $\mathrm{n}_{\mathrm{p}}\left(\times 10^{16} \mathrm{~cm}^{-3}\right)$

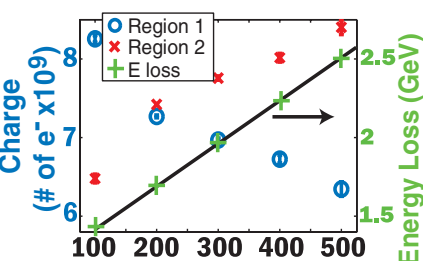

(b) CTR Energy (AU)
FIG. 3 (color). (a) Integrated $e^{+}$yield in the 4-20 MeV energy range vs plasma density. The black diamonds show the variation in yield with $\pm 0.5 \mu \mathrm{m}$ changes to $\sigma_{x, y}$. (b) The charge in region (1) of the electron spectrometer image (blue circles) and the charge in region (2) (red x's) vs $U_{\mathrm{CTR}}\left(\sim 1 / \sigma_{z}\right)$. At $U_{\mathrm{CTR}}=100$, there has already been charge transfer from region (1) to region (2). Also plotted is the mean beam energy loss for the radiating electrons in traversing an $11 \mathrm{~cm}$ plasma with $n_{p}=$ $1 \times 10^{17} \mathrm{~cm}^{-3}$ (green crosses).

photon energy can produce $e^{+}-e^{-}$pairs and thus the scaling of Eq. (1) also applies to the total $e^{+}$yield. Clearly, this $n_{p}^{2}$ scaling of Eq. (1) is not evident in Fig. 2(b). This is due partly to the density-dependent focusing of the beam at the Li-column entrance and partly to the increased energy loss to the wakefield, both being the result of utilizing much higher densities in this work. This strong interplay between the beam and plasma parameters makes Eq. (1) unsuitable as a design tool for predicting the performance of a plasmawiggler-based $e^{+}$source. Therefore, to calculate the photon spectrum that can reasonably predict the $e^{+}$yield, three critical parameters of the electron beam within the plasma must be determined. These are the number of electrons $N_{b}$ that participate in betatron oscillations, the mean energy $\gamma_{b}$ of the beam electrons, and the maximum spot sizes $\sigma_{x, y}$ of the beam in the plasma (the saddle points for $\theta=0$ ). Here, we experimentally estimate $N_{b}$ and $\gamma_{b}$ as described below and calculate $\sigma_{x, y}$ by using the beam envelope model, as was described earlier in connection with Fig. 2(a). To elucidate the beam physics and study how such a $e^{+}$source might be optimized, these quantities are studied over a range of plasma densities and plasma lengths $L_{p}$ as well as for different incoming bunch lengths.

We can obtain an estimate $N_{b}$ by measuring the number of electrons that lose energy. Figure 1(b) showed a typical electron energy spectrum. For analysis purposes, we have indicated two regions on this spectrum. Region (1), from $29-28 \mathrm{GeV}$, is the range of energies seen when no plasma is present (indicated by the dashed contour) and region (2), from $28-26 \mathrm{GeV}$, contains electrons that have definitely lost energy. As the beam enters the Li vapor, it begins to form a plasma only after the threshold for field ionization is reached. The transmitted electrons up to this point in the rising edge of the beam must therefore strike region (1) in the spectrum. Beyond the ionization threshold, beam electrons do work expelling the plasma electrons. Thus the electrons that strike region (2) reside in the ion column and give us the measure of $N_{b}$. As we make the bunch shorter, 

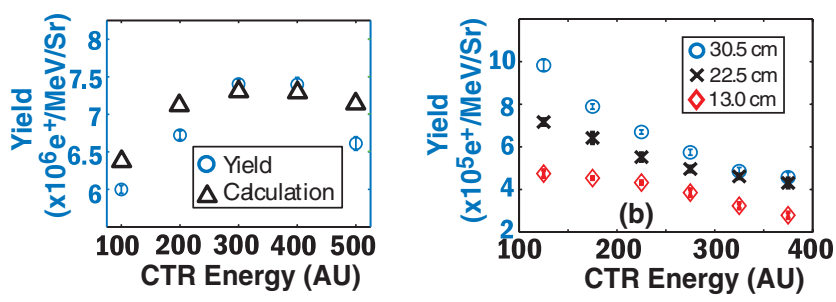

FIG. 4 (color). (a) The integrated measured and calculated $e^{+}$ yield in the 4-20 MeV energy range vs $U_{\mathrm{CTR}}$ for $n_{p}=1 \times$ $10^{17} \mathrm{~cm}^{-3}$ and $L_{p}=11 \mathrm{~cm}$. (b) Integrated $e^{+}$yield in the $27-$ $30 \mathrm{MeV}$ energy range vs $U_{\mathrm{CTR}}$ for 3 different plasma lengths with $n_{p}=2.7 \times 10^{17} \mathrm{~cm}^{-3}$. The CTR scale may be different between the two plots.

the ionization occurs earlier and charge is essentially transferred from region (1) to region (2). This transfer is clearly seen in Fig. 3(b), where we have plotted the absolute charge in each region versus $U_{\mathrm{CTR}} \sim 1 / \sigma_{z}$. We see, for example, that for $100 \leq U_{\mathrm{CTR}} \leq 300$ [the range of values used in Fig. 2(b)], $6.4 \times 10^{9} \lesssim N_{b} \lesssim 7.8 \times 10^{9}$ electrons; i.e., at most $\sim 0.46 N_{b o}$. For $\gamma_{b}$ at the plasma exit we take the mean energy of the electrons in region (2) of the spectra. The variation of the mean energy loss $\frac{1}{2} \times$ $\left(\gamma-\gamma_{b}\right) m c^{2}$ with $U_{\mathrm{CTR}}$ is also shown in Fig. 3(b) where $\gamma$ is the mean energy of the beam in the absence of Li. Note that this energy loss increases as $U_{\mathrm{CTR}}$ increases reaching nearly $2.6 \mathrm{GeV}$ at $U_{\mathrm{CTR}}=500\left(\sigma_{z} \sim 16 \mu \mathrm{m}\right)$. For computing the $\mathrm{x}$-ray spectrum, we assume that this net energy loss is distributed linearly along the saddle points. Using this recipe for determining the actual beam parameters, the calculated $e^{+}$spectra agree in both shape and absolute yield, as shown by the red curves in Fig. 2(b). Without these corrections, the calculated yield would be overestimated by a factor of $\sim 10$ for $n_{p}=1 \times 10^{17} \mathrm{~cm}^{-3}$ and even higher at higher densities.

The open circles in Fig. 4(a) show how the total yield in the energy range $4-20 \mathrm{MeV}$ varies as a function of $U_{\mathrm{CTR}}$ for $n_{p}=1 \times 10^{17} \mathrm{~cm}^{-3}$ and $L_{p}=11 \mathrm{~cm}$. The triangles in Fig. 4(a) show the calculated yield where the measured changes to $N_{b}$ and $\gamma_{b}$ shown in Fig. 3(b) were incorporated into the calculated photon spectra. Based on the good agreement of the calculated $e^{+}$yield to the experiment, we can infer that the rise in the $e^{+}$in Fig. 4(a) up to $U_{\mathrm{CTR}} \approx 300$ is dominated by the increase in $N_{b}$ as ionization occurs earlier while the subsequent fall of the yield is due to an increase in the energy loss to the wakefield when shorter bunches are used.

Up to this point we have presented data for $n_{p} \leq 1.0 \times$ $10^{17} \mathrm{~cm}^{-3}$ where the coupling of the SLAC electron beam to the plasma is relatively weak (on a per meter basis). When $n_{p}$ is increased to $2.7 \times 10^{17} \mathrm{~cm}^{-3}$, the $e^{+}$spectrum (not shown) shifts to higher energy, peaking at $\sim 30 \mathrm{MeV}$. In Fig. 4(b) we show the integrated $e^{+}$yield in this peakenergy range of $27-30 \mathrm{MeV}$ versus $U_{\text {CTR }}$ for three differ- ent Li vapor lengths. The $N_{b}$ versus $\gamma_{b}$ tradeoff seen in Fig. 4(a) is now not noticed due the higher coupling; the $\gamma_{b}$ variations dominate. When the wakefield loss is relatively small, for example, for the $U_{\mathrm{CTR}}$ bin of 125 in Fig. 4(b), the $e^{+}$yields increase from stepping $L_{p}$ from $13 \mathrm{~cm}$ to $22.5 \mathrm{~cm}$ and again from $22.5 \mathrm{~cm}$ to $30.5 \mathrm{~cm}$ is roughly in proportion to the relative increase in the plasma length. Although an ion column, and thus a wakefield, is a necessary condition for an effective plasma wiggler, the inevitable accumulation of energy loss with increasing plasma length eventually alters the betatron $\mathrm{x}$-ray spectrum enough to essentially shut off the production of high-energy positrons. This can be seen, for example, in the $U_{\mathrm{CTR}}=375$ bin of Fig. 4(b), where the average energy loss to the wake is larger than that of the 125 bin. Here there is a smaller increase in yield as the plasma length is increased from $13 \mathrm{~cm}$ to $22.5 \mathrm{~cm}$, but essentially no further yield increase when the length is increased to $30.5 \mathrm{~cm}$.

In conclusion, we have investigated a new scheme for generating positrons based on betatron $\mathrm{x}$ rays with energies of tens of $\mathrm{MeV}$ emitted in a plasma wiggler that may be useful for supplying $e^{+} \mathrm{s}$ for future accelerators. The measured $e^{+}$spectra are in excellent agreement with the calculated $\mathrm{x}$-ray spectra once the effect of the plasma in modifying the beam parameters is taken into account.

This work was funded with DOE Grants No. DE-FG0292ER40727, No. DE-FG03-92ER40745, No. DE-AC0276 SF00515 (SLAC), and NSF Grant: No. PHY-0406758.

[1] "SLC Design Handbook," edited by R. Erickson (1984), Chap. 5, pp. 15-79 (unpublished).

[2] G. Alexander et al., Report No. SLAC-TN-04-018, 2003 (unpublished).

[3] "Proceedings of the Workshop on New Kinds of Positron Sources for Linear Colliders," SLAC-R-502, 1997 (unpublished).

[4] K. Flottmann, in Handbook of Accelerator Physics and Engineering, edited by A. Chao and M. Tigner, Conversion of Undulator Radiation (World Scientific, Singapore, 1998), p. 433.

[5] D. H. Whittum, A.M. Sessler, and J.M. Dawson, Phys. Rev. Lett. 64, 2511 (1990).

[6] S. Wang et al., Phys. Rev. Lett. 88, 135004 (2002).

[7] E. Esarey et al., Phys. Rev. E 65, 056505 (2002).

[8] D. L. Bruhwiler et al., Phys. Plasmas, 10, 2022 (2003).

[9] C. O'Connell et al., Phys. Rev. ST Accel. Beams (to be published).

[10] C. E. Clayton et al., Phys. Rev. Lett. 88, 154801 (2002).

[11] C. Joshi et al., Phys. Plasmas 9, 1845 (2002).

[12] M. J. Hogan et al., Phys. Rev. Lett. 95, 054802 (2005).

[13] C. D. Barnes, Ph.D. thesis, Stanford University, 2006.

[14] K. A. Marsh et al., Proceedings of the 2005 Particle Accelerator Conference (IEEE, Piscataway, NJ, 2005).

[15] I. Kostyukov et al., Phys. Plasmas 10, 4818 (2003). 